\title{
The bounds estimate of sub-band operators for multi-band wavelets
}

\author{
Qingyun Zou ${ }^{1 *}$, Guoqiu Wang ${ }^{2}$ and Qian Cao ${ }^{1,2}$
}

${ }^{*}$ Correspondence: qyzou2000@sina.com

${ }^{1}$ Hunan Province Cooperative Innovation Center for The Construction Development of Dongting Lake Ecological Economic Zone, Hunan University of Arts and Science, Changde, P.R. China Full list of author information is available at the end of the article

\begin{abstract}
A concept of the sub-band operator of multi-band wavelets is introduced, the theory of d-circular matrices is developed and the upper bound and the lower bound of the norm of the sub-band operator are obtained. Examples are provided to illustrate the results proposed in this paper.
\end{abstract}

MSC: 42C40; 42A16

Keywords: Multi-band wavelets; Circular matrix; Biorthogonality; Sub-band operators; Bounds estimate

\section{Introduction}

During the last three decades, the theory of frames, which generalize the notion of bases by allowing redundancy yet still providing a reconstruction formula, has been growing rapidly, since several new applications such as nonlinear sparse approximation (e.g., image compression), coarse quantization, data transmission with erasures, and wireless communication, have been developed [1-7]. As a special class of frames, the multi-band wavelets have attracted considerable attention due to their richer parameter space, to give better energy compaction than 2-band wavelets [8-16].

Let $H$ be a separable Hilbert space, and $E$ an indexing set. A sequence $\left\{f_{l}\right\}_{l \in E}$ is called a frame in $H$ if there exist constants $0<A \leq B<+\infty$ such that

$$
A\|f\|^{2} \leq \sum_{l \in E}\left|\left\langle f, f_{l}\right\rangle\right|^{2} \leq B\|f\|^{2}, \quad \forall f \in H,
$$

where $A$ and $B$ are called lower and upper frame bounds, respectively. If $A=B$, the frame is called tight frame.

When $H=L^{2}(R)$, a wavelet $\psi(x) \in H$ gives rise to a classical wavelet frame $\left\{a^{-j / 2} \psi\left(a^{-j} x-\right.\right.$ $b k), j, k \in Z$ \} with parameters $a>1, b>0$. Chui and Shi [17] established the relationship between the parameters and the frame bounds,

$$
A \leq \frac{1}{2 b \ln a} \int_{-\infty}^{+\infty} \frac{|\widehat{\psi}(\omega)|^{2}}{|\omega|} d \omega \leq B
$$

Equation (1.2) shows that the energy of a biorthogonal wavelet transform is controllable although it is not conservative. Moreover, $B-A$ or $\widetilde{B}-\widetilde{A}$ are smaller, the performance of

(c) The Author(s) 2018. This article is distributed under the terms of the Creative Commons Attribution 4.0 International License (http://creativecommons.org/licenses/by/4.0/), which permits unrestricted use, distribution, and reproduction in any medium, provided you give appropriate credit to the original author(s) and the source, provide a link to the Creative Commons license, and indicate if changes were made. 
a biorthogonal wavelet transform may be better, i.e., the energy is amplified in some cases and decreased in other cases. The classical biorthogonal wavelets are not tight frames, so obtaining the exact values of their bounds is difficult. Instead of estimating the bounds in (1.2), we can try to obtain the upper bound and the lower bound of the norm of the sub-band operator.

This paper is organized as follows. In Sect. 2, we define the sub-band operator, and obtain the limit form of the norm of the sub-band operator. We present a method for computing the upper bound and the lower bound of the norm of the sub-band operator based on the theory of circular matrix. Section 3 gives some examples to illustrate the results proposed in this paper.

\section{Sub-band operator and $d$-circular matrix}

Recall the sub-band coding scheme or Mallat algorithm associated to a $d$-band real biorthogonal wavelets. There are $2 d$ filters $h=\left(h_{n}\right)_{n \in Z}, g^{i}=\left(g_{n}^{i}\right)_{n \in Z}(i=1,2, \ldots, d-1)$, $\widetilde{h}=\left(\widetilde{h}_{n}\right)_{n \in Z}$ and $\widetilde{g}^{i}=\left(\widetilde{g}_{n}^{i}\right)_{n \in Z}(i=1,2, \ldots, d-1),\left\{h, g^{1}, g^{2}, \ldots, g^{d-1}\right\}$ are used for decomposition and $\left\{\tilde{h}, \widetilde{g}^{1}, \widetilde{g}^{2}, \ldots, \widetilde{g}^{d-1}\right\}$ for reconstruction. Starting from a data sequence $x=\left(x_{n}\right)_{n \in Z}$, we convolve with $\left\{h, g^{1}, g^{2}, \ldots, g^{d-1}\right\}$,

$$
\begin{aligned}
& c_{n}=\sum_{k} h_{d n-k} x_{k}, \\
& p_{n}^{i}=\sum_{k} g_{d n-k}^{i} x_{k}, \quad i=1,2, \ldots, d-1 .
\end{aligned}
$$

The reconstruction operation is

$$
\tilde{x}_{k}=\sum_{n}\left(\tilde{h}_{d n-k} c_{n}+\sum_{i=1}^{d-1} \widetilde{g}_{d n-k}^{i} p_{n}^{i}\right) .
$$

The constraint conditions for biorthogonal $d$-band filter banks with perfect reconstruction property are:

a. the low-pass and high-pass condition

$$
\sum h_{k}=\sum \widetilde{h}_{k}=\sqrt{d}, \quad \sum g_{k}^{i}=\sum \widetilde{g}_{k}^{i}=0, \quad i=1,2, \ldots, d-1
$$

b. the biorthogonal condition

$$
\sum_{k} h_{k} \widetilde{h}_{k+d j}=\delta_{j}, \quad h_{k} \widetilde{g}_{k+d j}^{i}=g_{k}^{i} \widetilde{h}_{k+d j}=0, \quad g_{k}^{i} \widetilde{g}_{k+d j}^{l}=\delta_{i-l} \delta_{j},
$$

where $\delta_{j}$ denotes the Dirac sequence such that $\delta_{j}=1$ for $j=0$ otherwise $\delta_{j}=0$;

c. the perfect reconstruction condition $x=\widetilde{x}$.

In order to define (2.1) and (2.2) as operators, namely, sub-band operators, we assume that the input signal $x \in l^{2}(-\infty,+\infty)$. Now consider the separable Hilbert space $l^{2}(-\infty,+\infty)$. Define

$$
(x, y)=\sum_{n=-\infty}^{+\infty} x_{i} \bar{y}_{i}
$$


as the usual inner product on $l^{2}(-\infty,+\infty)$, where $x, y \in l^{2}(-\infty,+\infty)$, and $\bar{y}_{i}$ denotes the conjugate of the complex number $y_{i}$.

Definition 2.1 The operator

$$
T: T x=y
$$

is called a sub-band operator, where $x \in l^{2}(-\infty,+\infty), y=\left(\ldots, c_{0}, p_{0}^{1}, p_{0}^{2}, \ldots, p_{0}^{d-1}, c_{1}, p_{1}^{1}\right.$, $\left.p_{1}^{2}, \ldots, p_{1}^{d-1}, \ldots\right), c_{n}$ and $p_{n}^{i}, i=1,2, \ldots, d-1$ are defined as in (2.1).

Throughout this paper, we assume $h, g^{1}, g^{2}, \ldots, g^{d-1}, \widetilde{h}, \widetilde{g}^{1}, \widetilde{g}^{2}, \ldots, \widetilde{g}^{d-1}$ have only finitely many nonzero elements.

Theorem 2.1 If the d-band biorthogonal wavelets determined by the filters $h, g^{1}, g^{2}, \ldots$, $g^{d-1}, \widetilde{h}, \widetilde{g}^{1}, \widetilde{g}^{2}, \ldots, \widetilde{g}^{d-1}$ have the perfect reconstruction property, then the sub-band operator $T$ is a bounded linear operator and reversible on $l^{2}(-\infty,+\infty)$.

The proof of Theorem 2.1 is trivial.

As is well known, a bounded linear operator on $l^{2}(-\infty,+\infty)$ can be expressed by an infinite-dimensional matrix. Using matrix notations, we have a more helpful expression of the operator $T$. Let

$$
s_{n, k}=\left[\begin{array}{c}
h_{k-d n} \\
g_{k-d n}^{1} \\
g_{k-d n}^{2} \\
\vdots \\
g_{k-d n}^{d-1}
\end{array}\right], \quad \widetilde{s}_{n, k}=\left[\begin{array}{c}
\widetilde{h}_{k-d n} \\
\widetilde{g}_{k-d n}^{1} \\
\widetilde{g}_{k-d n}^{2} \\
\vdots \\
\widetilde{g}_{k-d n}^{d-1}
\end{array}\right] .
$$

Then $A=\left[s_{n, k}\right]$ and $\widetilde{A}=\left[\widetilde{s}_{n, k}\right](-\infty<n, k<+\infty)$ are infinite block circular matrices along four directions (up, down, left and right). At this time,

$$
y=A x,
$$

where $x$ and $y$ are doubly infinite column vectors to fit the matrix operation. Hence $T$ can be viewed as an infinite matrix $A$, i.e., $T=A$. If the sub-band decomposition has the perfect reconstruction condition, then it is obvious that $A$ and $\widetilde{A}$ should satisfy

$$
A \widetilde{A}^{*}=\widetilde{A}^{*} A=I
$$

where $I$ denotes the infinite identity matrix and $A^{*}$ denotes the transpose of complex conjugate of $A$. Thus, $T^{-1}=\widetilde{A}^{*}$.

Since $(A x, y)=\left(x, A^{*} y\right)$, the adjoint operator of $T$ is $T^{*}=A^{*}$. Let $Q=T^{*} T$, we have the following lemma. 
Lemma 2.1 Retaining the definitions and notations as above, we have

(1) $\|Q\|=\|T\|^{2}$;

(2) $\quad T^{-1}=\widetilde{T}^{*}=\widetilde{A}^{*}, \quad \widetilde{T}^{-1}=T^{*}=A^{*}$;

(3) $\|Q\|=\sup _{\|x\|=1}\{|(Q x, x)|\}$.

Proof Items (1) and (2) are trivial according to the operator theory [18]. Item (3) follows the fact that $Q$ is a self-adjoint operator due to $Q^{*}=Q$.

$Q=T^{*} T$ is called a frame operator in general [2]. Let $v_{n}$ and $\widetilde{v}_{n}$ denote the $n$th rows of $A$ and $\tilde{A}$, respectively. Clearly, $\left(v_{j}, \widetilde{v}_{k}\right)=\delta_{j-k}$, where $\delta_{j}$ is the Dirac sequence, i.e., $\delta_{j}=1$ for $j=0$ otherwise $\delta_{j}=0$. Therefore, $\left\{v_{n}\right\}$ and $\left\{\widetilde{v}_{n}\right\}$ are dual biorthogonal bases in $l^{2}(-\infty,+\infty)$. Let $e_{n} \in l^{2}(-\infty,+\infty)$, its $n$th component be 1 and otherwise be 0 . Then $T \widetilde{v}_{n}=e_{n}=\widetilde{T} v_{n}$. For an arbitrary $x \in l^{2}(-\infty,+\infty)$,

$$
\begin{aligned}
& x=\sum_{n=-\infty}^{+\infty}\left(x, v_{n}\right) \widetilde{v}_{n}=\sum_{n=-\infty}^{+\infty}\left(x, \widetilde{v}_{n}\right) v_{n}, \\
& Q x=\sum_{n=-\infty}^{+\infty}\left(x, v_{n}\right) Q \widetilde{v}_{n}=\sum_{n=-\infty}^{+\infty}\left(x, v_{n}\right) T^{*} e_{n}=\sum_{n=-\infty}^{+\infty}\left(x, v_{n}\right) T^{*} \widetilde{T} v_{n}=\sum_{n=-\infty}^{+\infty}\left(x, v_{n}\right) v_{n} .
\end{aligned}
$$

Let $m$ and $M$ denote the lower bound and the upper bound of $\|T\|$, respectively.

$$
\begin{aligned}
& T x=\sum_{n=-\infty}^{+\infty}\left(x, v_{n}\right) T \widetilde{v}_{n}=\sum_{n=-\infty}^{+\infty}\left(x, v_{n}\right) e_{n}, \\
& \|T x\|^{2}=\sum_{n=-\infty}^{+\infty}\left|\left(x, v_{n}\right)\right|^{2} .
\end{aligned}
$$

Then

$$
m^{2}\|x\|^{2} \leq \sum_{n=-\infty}^{+\infty}\left|\left(x, v_{n}\right)\right|^{2} \leq M^{2}\|x\|^{2}
$$

Similarly,

$$
\widetilde{m}^{2}\|x\|^{2} \leq \sum_{n=-\infty}^{+\infty}\left|\left(x, \widetilde{v}_{n}\right)\right|^{2} \leq \widetilde{M}^{2}\|x\|^{2}
$$

where $\widetilde{m}$ and $\widetilde{M}$ are the lower bound and the upper bound of $\|\widetilde{T}\|$, respectively. Therefore, (2.4) and (2.5) are the counterparts of $(1.1)$ in $l^{2}(-\infty,+\infty)$. 
Now we define a finite matrix $A_{n}$ as the partial matrix of the infinite matrix $A$, its row index and column index are finite with the following form:

$$
A_{n}=\left[\begin{array}{cccccccccc}
h_{0} & h_{1} & h_{2} & \ldots & h_{d-1} & \ldots & 0 & 0 & 0 & 0 \\
g_{0}^{1} & g_{1}^{1} & g_{2}^{1} & \ldots & g_{d-1}^{1} & \ldots & 0 & 0 & 0 & 0 \\
g_{0}^{2} & g_{1}^{2} & g_{2}^{2} & \ldots & g_{d-1}^{2} & \ldots & 0 & 0 & 0 & 0 \\
g_{0}^{d-1} & g_{1}^{d-1} & g_{2}^{d-1} & \ldots & g_{d-1}^{d-1} & \ldots & 0 & 0 & 0 & 0 \\
h_{-d} & \ldots & h_{-3} & h_{-2} & h_{-1} & \ldots & 0 & 0 & 0 & 0 \\
g_{-d}^{1} & \ldots & g_{-3}^{1} & g_{-2}^{1} & g_{-1}^{1} & \ldots & 0 & 0 & 0 & 0 \\
g_{-d}^{2} & \ldots & g_{-3}^{2} & g_{-2}^{2} & g_{-1}^{2} & \ldots & 0 & 0 & 0 & 0 \\
g_{-d}^{d-1} & \ldots & g_{-3}^{d-1} & g_{-2}^{d-1} & g_{-1}^{d-1} & \ldots & 0 & 0 & 0 & 0 \\
\ldots & \ldots & \ldots & \ldots & \ldots & \ldots & \ldots & \ldots & \ldots & \ldots \\
0 & 0 & 0 & 0 & \ldots & h_{0} & h_{1} & h_{2} & \ldots & h_{d-1} \\
0 & 0 & 0 & 0 & \ldots & g_{0}^{1} & g_{1}^{1} & g_{2}^{1} & \ldots & g_{d-1}^{1} \\
0 & 0 & 0 & 0 & \ldots & g_{0}^{2} & g_{1}^{2} & g_{2}^{2} & \ldots & g_{d-1}^{2} \\
0 & 0 & 0 & 0 & \ldots & g_{0}^{d-1} & g_{1}^{d-1} & g_{2}^{d-1} & \ldots & g_{d-1}^{d-1} \\
0 & 0 & 0 & 0 & \ldots & h_{-d} & \ldots & h_{-3} & h_{-2} & h_{-1} \\
0 & 0 & 0 & 0 & \ldots & g_{-d}^{1} & \ldots & g_{-3}^{1} & g_{-2}^{1} & g_{-1}^{1} \\
0 & 0 & 0 & 0 & \ldots & g_{-d}^{2} & \ldots & g_{-3}^{2} & g_{-2}^{2} & g_{-1}^{2} \\
0 & 0 & 0 & 0 & \ldots & g_{-d}^{d-1} & \ldots & g_{-3}^{d-1} & g_{-2}^{d-1} & g_{-1}^{d-1}
\end{array}\right]
$$

Theorem 2.2 Let $Q_{n}=A_{n}^{*} A_{n}$ and $\left\{\tau_{1}^{(n)}, \tau_{2}^{(n)}, \ldots, \tau_{d n}^{(n)}\right\}$ be all eigenvalues of $Q_{n}$. Then

$$
\|Q\|=\lim _{n \rightarrow \infty} \max \left\{\tau_{1}^{(n)}, \tau_{2}^{(n)}, \ldots, \tau_{d n}^{(n)}\right\}
$$

Proof Since $Q_{n}$ is a positive operator, it has $d n$ positive real eigenvalues, and $(Q x, x) \geq 0$.

From Lemma 2.1, we have

$$
\|Q\|=\sup _{\|x\|=1}\{|(Q x, x)|\}=\sup _{\|x\|=1}\{(Q x, x)\}
$$

Hence, there exists an $x^{(m)} \in l^{2}(-\infty,+\infty)$, such that $\left\|x^{(m)}\right\|=1$ and $\|Q\|=$ $\lim _{m \rightarrow \infty}\left(Q x^{(m)}, x^{(m)}\right)$. Let $d n$-dimensional vector $[x]_{n}^{(m)}$ be the finite part of $x^{(m)}$.

On one hand, clearly, $\left\|[x]_{n}^{(m)}\right\| \leq\left\|x^{(m)}\right\|$ for an arbitrary $n$. Let $[y]_{n}$ be a $d n$-dimensional vector. Then

$$
\|Q\|=\lim _{m \rightarrow \infty}\left(Q x^{(m)}, x^{(m)}\right)=\lim _{m \rightarrow \infty} \lim _{n \rightarrow \infty}\left(Q_{n}[x]_{n}^{(m)},[x]_{n}^{(m)}\right)
$$

Since $Q_{n}$ is a finite-dimensional self-adjoint compact operator, we have

$$
\sup _{\left\|[y]_{n}\right\| \leq 1}\left\{\left(Q_{n}[y]_{n},[y]_{n}\right)\right\}=\max \left\{\tau_{1}^{(n)}, \tau_{2}^{(n)}, \ldots, \tau_{d n}^{(n)}\right\}
$$

Hence,

$$
\|Q\| \leq \lim _{n \rightarrow \infty} \max \left\{\tau_{1}^{(n)}, \tau_{2}^{(n)}, \ldots, \tau_{d n}^{(n)}\right\}
$$


On the other hand, for every sufficiently large $n$, there exists a $d n$-dimensional vector $[y]_{n}$, such that $\left\|[y]_{n}\right\|=1$, and

$$
\sup _{\|y\|=1}\left\{\left(Q_{n}[y],[y]\right)\right\}=\left(Q_{n}[y]_{n},[y]_{n}\right)=\max \left\{\tau_{1}^{(n)}, \tau_{2}^{(n)}, \ldots, \tau_{d n}^{(n)}\right\}
$$

Extend $[y]_{n}$ to $y^{(n)} \in l^{2}(-\infty,+\infty)$ by appending 0 s to the tuples which are not defined by $[y]_{n}$. Clearly, $\left\|[y]_{n}\right\|=\left\|y^{(n)}\right\|=1$, and

$$
\|Q\|=\sup _{\|x\|=1}\{(Q x, x)\} \geq\left(Q y^{(n)}, y^{(n)}\right)=\left(Q[y]_{n},[y]_{n}\right)=\max \left\{\tau_{1}^{(n)}, \tau_{2}^{(n)}, \ldots, \tau_{d n}^{(n)}\right\} .
$$

It implies that

$$
\|Q\| \geq \lim _{n \rightarrow \infty} \max \left\{\tau_{1}^{(n)}, \tau_{2}^{(n)}, \ldots, \tau_{d n}^{(n)}\right\}
$$

Hence, $\|Q\|=\lim _{n \rightarrow \infty} \max \left\{\tau_{1}^{(n)}, \tau_{2}^{(n)}, \ldots, \tau_{d n}^{(n)}\right\}$. The proof is complete.

Theoretically, Theorem 2.2 gives an exact value for $\|Q\|$ and $\|T\|=\sqrt{\|Q\|}$ is used to compute the norm of $T$. However, the eigenvalues are not easy to compute for generalized block Toeplitz matrices. We shall use the theory of circular matrix to compute the norm of $Q$. The block circular matrix is defined as follows [19]:

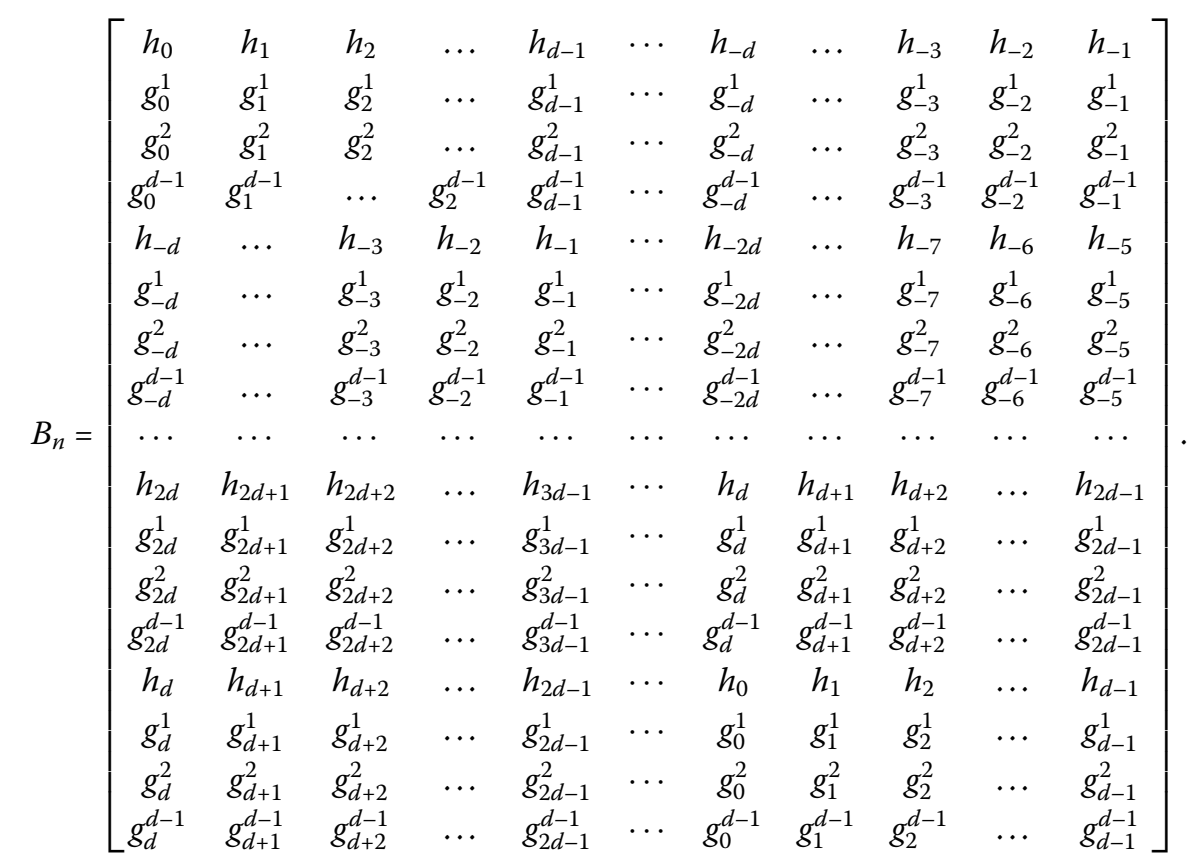

Clearly, $A_{n}$ is different from $B_{n}$. Let $C_{n}=B_{n}-A_{n}$, then only finitely many (fixed) elements in $C_{n}$ are not 0 no matter how large the dimension $4 n$ is. We have

$$
A_{n}^{*} A_{n}=B_{n}^{*} B_{n}-C_{n}^{*} B_{n}-B_{n}^{*} C_{n}+C_{n}^{*} C_{n} .
$$

We have Theorem 2.3. 
Theorem 2.3 Let $P_{n}=B_{n}^{*} B_{n}$ and $\left\{\lambda_{1}^{(n)}, \lambda_{2}^{(n)}, \ldots, \lambda_{d n}^{(n)}\right\}$ be all eigenvalues of $P_{n}$. Then

$$
\|Q\|=\lim _{n \rightarrow \infty} \max \left\{\lambda_{1}^{(n)}, \lambda_{2}^{(n)}, \ldots, \lambda_{d n}^{(n)}\right\}
$$

Proof Let $[x]_{n}$ be a $d n$-dimensional vector. We first prove that for $\left\|[x]_{n}\right\|=1$, $\lim _{n \rightarrow \infty}\left|\left(C_{n}^{*} B_{n}[x]_{n},[x]_{n}\right)\right|=0, \lim _{n \rightarrow \infty}\left|\left(B_{n}^{*} C_{n}[x]_{n},[x]_{n}\right)\right|=0, \lim _{n \rightarrow \infty}\left|\left(C_{n}^{*} C_{n}[x]_{n},[x]_{n}\right)\right|=0$.

In fact, the operator $B_{n}$ is bounded. For $\left\|[x]_{n}\right\|=1$, there exists a positive real number $M$ such that

$$
\begin{aligned}
\left|\left(C_{n}^{*} B_{n}[x]_{n},[x]_{n}\right)\right| & =\left|\left(B_{n}[x]_{n}, C_{n}[x]_{n}\right)\right| \\
& \leq\left\|B_{n}[x]_{n}\right\|\left\|C_{n}[x]_{n}\right\| \leq M\left\|C_{n}[x]_{n}\right\| .
\end{aligned}
$$

Note that $C_{n}[x]_{n}$ only contains $d n$ nonzero components.

Hence $\lim _{n \rightarrow \infty}\left\|C_{n}[x]_{n}\right\|=0$, i.e., $\lim _{n \rightarrow \infty}\left|\left(C_{n}^{*} C_{n}[x]_{n},[x]_{n}\right)\right|=0$.

Similarly, we can verify $\lim _{n \rightarrow \infty}\left|\left(B_{n}^{*} C_{n}[x]_{n},[x]_{n}\right)\right|=0$ and $\lim _{n \rightarrow \infty}\left|\left(C_{n}^{*} C_{n}[x]_{n},[x]_{n}\right)\right|=0$.

It follows from (2.6) that

$$
\left(Q_{n}[x]_{n},[x]_{n}\right)=\left(P_{n}[x]_{n},[x]_{n}\right)+\left(R_{n}[x]_{n},[x]_{n}\right),
$$

where $R_{n}=-C_{n}^{*} B_{n}-B_{n}^{*} C_{n}+C_{n}^{*} C_{n}$ and $\lim _{n \rightarrow \infty}\left|\left(R_{n},[x]_{n}\right)\right|=0$.

It implies that

$$
\lim _{n \rightarrow \infty} \sup _{\left\|[x]_{n}\right\|=1}\left\{\left(Q_{n}[x]_{n},[x]_{n}\right)\right\}=\lim _{n \rightarrow \infty} \sup _{\left\|[x]_{n}\right\|=1}\left\{\left(P_{n}[x]_{n},[x]_{n}\right)\right\}
$$

By Theorem 2.2, we have

$$
\|Q\|=\lim _{n \rightarrow \infty} \max \left\{\tau_{1}^{(n)}, \tau_{2}^{(n)}, \ldots, \tau_{d n}^{(n)}\right\}=\lim _{n \rightarrow \infty} \max \left\{\lambda_{1}^{(n)}, \lambda_{2}^{(n)}, \ldots, \lambda_{d n}^{(n)}\right\}
$$

The proof is complete.

A so-called $d$-circular matrix [20], which is generated by the filters $h, g^{1}, g^{2}, \ldots, g^{d-1}$, is denoted as $M_{n}$. For $d=4, M_{3}$ is as follows:

$$
M_{3}=\left[\begin{array}{cccccccccccc}
h_{0} & h_{1} & h_{2} & h_{3} & 0 & 0 & 0 & 0 & h_{-4} & h_{-3} & h_{-2} & h_{-1} \\
h_{-4} & h_{-3} & h_{-2} & h_{-1} & h_{0} & h_{1} & h_{2} & h_{3} & 0 & 0 & 0 & 0 \\
0 & 0 & 0 & 0 & h_{-4} & h_{-3} & h_{-2} & h_{-1} & h_{0} & h_{1} & h_{2} & h_{3} \\
g_{0}^{1} & g_{1}^{1} & g_{2}^{1} & g_{3}^{1} & 0 & 0 & 0 & 0 & g_{-4}^{1} & g_{-3}^{1} & g_{-2}^{1} & g_{-1}^{1} \\
g_{-4}^{1} & g_{-3}^{1} & g_{-2}^{1} & g_{-1}^{1} & g_{0}^{1} & g_{1}^{1} & g_{2}^{1} & g_{3}^{1} & 0 & 0 & 0 & 0 \\
0 & 0 & 0 & 0 & g_{-4}^{1} & g_{-3}^{1} & g_{-2}^{1} & g_{-1}^{1} & g_{0}^{1} & g_{1}^{1} & g_{2}^{1} & g_{3}^{1} \\
g_{0}^{2} & g_{1}^{2} & g_{2}^{2} & g_{3}^{2} & 0 & 0 & 0 & 0 & g_{-4}^{2} & g_{-3}^{2} & g_{-2}^{2} & g_{-1}^{2} \\
g_{-4}^{2} & g_{-3}^{2} & g_{-2}^{2} & g_{-1}^{2} & g_{0}^{2} & g_{1}^{2} & g_{2}^{2} & g_{3}^{2} & 0 & 0 & 0 & 0 \\
0 & 0 & 0 & 0 & g_{-4}^{2} & g_{-3}^{2} & g_{-2}^{2} & g_{-1}^{2} & g_{0}^{2} & g_{1}^{2} & g_{2}^{2} & g_{3}^{2} \\
g_{0}^{3} & g_{1}^{3} & g_{2}^{3} & g_{3}^{3} & 0 & 0 & 0 & 0 & g_{-4}^{3} & g_{-3}^{3} & g_{-2}^{3} & g_{-1}^{3} \\
g_{-4}^{3} & g_{-3}^{3} & g_{-2}^{3} & g_{-1}^{3} & g_{0}^{3} & g_{1}^{3} & g_{2}^{3} & g_{3}^{3} & 0 & 0 & 0 & 0 \\
0 & 0 & 0 & 0 & g_{-4}^{3} & g_{-3}^{3} & g_{-2}^{3} & g_{-1}^{3} & g_{0}^{3} & g_{1}^{3} & g_{2}^{3} & g_{3}^{3}
\end{array}\right] .
$$


Clearly, $B_{n}$ and $M_{n}$ are not so different. One can be obtained by exchanging the places of some rows of another, i.e., there exists an orthonormal matrix $E_{n}$ such that $M_{n}=E_{n} B_{n}$. The purpose is to facilitate the calculation of the eigenvalues.

Similarly, let $\widetilde{M}_{n}$ be a $d$-circular matrix generated by $\widetilde{h}, \widetilde{g}^{1}, \widetilde{g}^{2}, \ldots, \widetilde{g}^{d-1}$. The perfect reconstruction condition is that there exists an integer $N_{0}$, such that, for all $n \geq N_{0}$ (in what follows, $n$ sufficiently large is in this sense),

$$
M_{n} \widetilde{M}_{n}^{*}=I_{d n}
$$

where $I_{d n}$ is a $d n \times d n$ identity matrix.

Theorem 2.4 Let $M_{n}$ be a d-circular matrix generated by $h, g^{1}, g^{2}, \ldots, g^{d-1}$. For sufficiently large $n$, then

$$
\max \left\{\lambda_{1}, \lambda_{2}, \ldots, \lambda_{d n}\right\} \leq \max \left\{C_{0}, C_{1}, \ldots, C_{d-1}\right\}
$$

where

$$
\begin{aligned}
& C_{0}=\sum_{j}\left|\sum_{i} h_{i} h_{i+d j}\right|+\sum_{n=1}^{d-1} \sum_{j}\left|\sum_{i} h_{i} g_{i+d j}^{n}\right|, \\
& C_{k}=\sum_{j}\left|\sum_{i} g_{i}^{k} h_{i+d j}\right|+\sum_{n=1}^{d-1} \sum_{j}\left|\sum_{i} g_{i}^{k} g_{i+d j}^{n}\right|, \quad 1 \leq k \leq d-1 .
\end{aligned}
$$

Proof Define

$$
\left\|A_{d n}\right\|_{\infty}=\max _{1 \leq i \leq d n} \sum_{j=1}^{d n}\left|a_{i, j}\right| .
$$

Since $\left\|A_{d n} B_{d n}\right\|_{\infty} \leq\left\|A_{d n}\right\|_{\infty} \times\left\|B_{d n}\right\|_{\infty},\|\cdot\|_{\infty}$ is a compatible matrix norm. Note that $A_{d n}=M_{n} M_{n}^{T}$ positive definite matrices, and all of the eigenvalues of $M_{n} M_{n}^{T}$ are positive. According to the theory of matrices [19], we have

$$
\left|\lambda_{i}\right| \leq\left\|A_{d n}\right\|_{\infty}=\max _{1 \leq i \leq d n} \sum_{j=1}^{d n}\left|a_{i, j}\right|, \quad i=1,2, \ldots, d n .
$$

Note that the sub-matrices of $M_{n} M_{n}^{T}$ such as $H H^{T}, H G_{1}^{T}, H G_{2}^{T}, \ldots, H G_{d-1}^{T}, \ldots$ are all 1circular matrices. Then

$$
\sum_{j=k n+1}^{k n+n}\left|a_{k n+1, j}\right|=\sum_{j=k n+1}^{k n+n}\left|a_{k n+2, j}\right|=\cdots=\sum_{j=k n+1}^{k n+n}\left|a_{k n+n, j}\right|, \quad 0 \leq k \leq d-1 .
$$

We have

$$
C_{0}=\sum_{j=1}^{d n}\left|a_{1, j}\right|=\sum_{j}\left|\sum_{i} h_{i} h_{i+d j}\right|+\sum_{n=1}^{d-1} \sum_{j}\left|\sum_{i} h_{i} g_{i+d j}^{n}\right|
$$


Firstly, we verify that, for $n \geq N_{0}$,

$$
\sum_{j=1}^{n}\left|a_{1, j}\right| \leq \sum_{j}\left|\sum_{i} h_{i} h_{i+d j}\right| .
$$

1. There exists $N_{0}^{*}$ : when $n \geq N_{0}^{*} \geq N_{0}$, we have

$$
H H^{T}=\left[\sum h_{i} h_{i}, \sum h_{i} h_{i+d}, \sum h_{i} h_{i+2 d}, \ldots, 0, \ldots, \sum h_{i} h_{i+d}\right] .
$$

Note that when the dimension increases, the nonzero elements of $H H^{T}$ are the same. Therefore

$$
\sum_{j=1}^{n}\left|a_{1, j}\right|=\sum_{j}\left|\sum_{i} h_{i} h_{i+d j}\right| .
$$

2. When $N_{0} \leq n \leq N_{0}^{*}$, the nonzero elements of $H H^{T}$ decrease.

It follows from $|a+b| \leq|a|+|b|$ that

$$
\sum_{j=1}^{n}\left|a_{1, j}\right| \leq \sum_{j}\left|\sum_{i} h_{i} h_{i+d j}\right|
$$

We obtain

$$
\sum_{j=1}^{n}\left|a_{1, j}\right| \leq \sum_{j}\left|\sum_{i} h_{i} h_{i+d j}\right|
$$

Similarly, (2.8) holds true.

Remark The right-hand side of (2.8) is only determined by the filters.

Theorem 2.5 Let $T$ be the sub-band operator of d-band wavelets. Then

$$
\begin{aligned}
& \frac{1}{\sqrt{\max \left\{\widetilde{C}_{0}, \widetilde{C}_{1}, \ldots, \widetilde{C}_{d-1}\right\}}} \leq\|T\| \leq \sqrt{\max \left\{C_{0}, C_{1}, \ldots, C_{d-1}\right\}} \\
& \frac{1}{\sqrt{\max \left\{C_{0}, C_{1}, \ldots, C_{d-1}\right\}}} \leq\left\|T^{-1}\right\| \leq \sqrt{\max \left\{\widetilde{C}_{0}, \widetilde{C}_{1}, \ldots, \widetilde{C}_{d-1}\right\}}
\end{aligned}
$$

where the filter bands are $\left\{h, g^{1}, g^{2}, \ldots, g^{d-1}\right\}$ and $\left\{\tilde{h}, \widetilde{g}^{1}, \widetilde{g}^{2}, \ldots, \widetilde{g}^{d-1}\right\}$, respectively,

$$
\begin{aligned}
& C_{0}=\sum_{j}\left|\sum_{i} h_{i} h_{i+d j}\right|+\sum_{n=1}^{d-1} \sum_{j}\left|\sum_{i} h_{i} g_{i+d j}^{n}\right|, \\
& C_{k}=\sum_{j}\left|\sum_{i} g_{i}^{k} h_{i+d j}\right|+\sum_{n=1}^{d-1} \sum_{j}\left|\sum_{i} g_{i}^{k} g_{i+d j}^{n}\right|, \quad 1 \leq k \leq d-1,
\end{aligned}
$$




$$
\begin{aligned}
& \widetilde{C}_{0}=\sum_{j}\left|\sum_{i} \widetilde{h}_{i} \widetilde{h}_{i+d j}\right|+\sum_{n=1}^{d-1} \sum_{j}\left|\sum_{i} \widetilde{h}_{i} \widetilde{g}_{i+d j}^{n}\right|, \\
& \widetilde{C}_{k}=\sum_{j}\left|\sum_{i} \widetilde{g}_{i}^{k} \widetilde{h}_{i+d j}\right|+\sum_{n=1}^{d-1} \sum_{j}\left|\sum_{i} \widetilde{g}_{i}^{k} \widetilde{g}_{i+d j}^{n}\right|, \quad 1 \leq k \leq d-1 .
\end{aligned}
$$

Proof By Theorem 2.3, we have

$$
\|T\|^{2}=\|Q\|=\lim _{n \rightarrow \infty} \max \left\{\lambda_{1}, \lambda_{2}, \ldots, \lambda_{d n}\right\}
$$

where $\left\{\lambda_{1}, \lambda_{2}, \ldots, \lambda_{d n}\right\}$ are all eigenvalues of $M_{n} M_{n}^{T}$.

According to Theorem 2.4, we have

$$
\|T\|=\sqrt{\|Q\|} \leq \sqrt{\max \left\{C_{0}, C_{1}, \ldots, C_{d-1}\right\}} .
$$

Similarly,

$$
\left\|T^{-1}\right\| \leq \sqrt{\max \left\{\widetilde{C}_{0}, \widetilde{C}_{1}, \ldots, \widetilde{C}_{d-1}\right\}}
$$

The proof is complete.

\section{Examples}

In this section, we present two examples to illustrate the proposed results.

Example 3.1 Let the lengths of scaling filters be $(15,9)$. Assume that the scaling symbols $\left(H_{0}(z), \widetilde{H}_{0}(z)\right)$ have the following form:

$$
\begin{aligned}
& H_{0}(z)=\left(\frac{1+z+z^{2}}{3}\right)^{5} Q(z), \\
& \widetilde{H}_{0}(z)=\left(\frac{1+z+z^{2}}{3}\right)^{3} \widetilde{Q}(z),
\end{aligned}
$$

where $(Q(z), \widetilde{Q}(z))$ are symmetric Laurent polynomials with degree $(4,2)$.

We can obtain the associated scaling filters as follows [21]:

$$
\begin{aligned}
h_{0} \approx & {[0.0302708750,0.0197271260,0.0109853080,-0.12261759,0.011382944,} \\
& 0.24928687,0.83207454,0.93777986], \\
\widetilde{h}_{0} \approx & {[-0.20140256,-0.090291448,0.13193077,1.0694718,1.1805829] }
\end{aligned}
$$

(whereas the other half is symmetric and so is skipped). Thus, the wavelet filters $h_{1}, h_{2}$ and $\widetilde{h}_{1}, \widetilde{h}_{2}$ can be obtained as follows:

$$
\begin{aligned}
& h_{1} \approx[0.032348717,0.021081228,0.011739358,-0.75426796,1.3781973], \\
& h_{2} \approx 4[-0.044296947,-0.028867731,-0.016075373,0.29594173,0],
\end{aligned}
$$



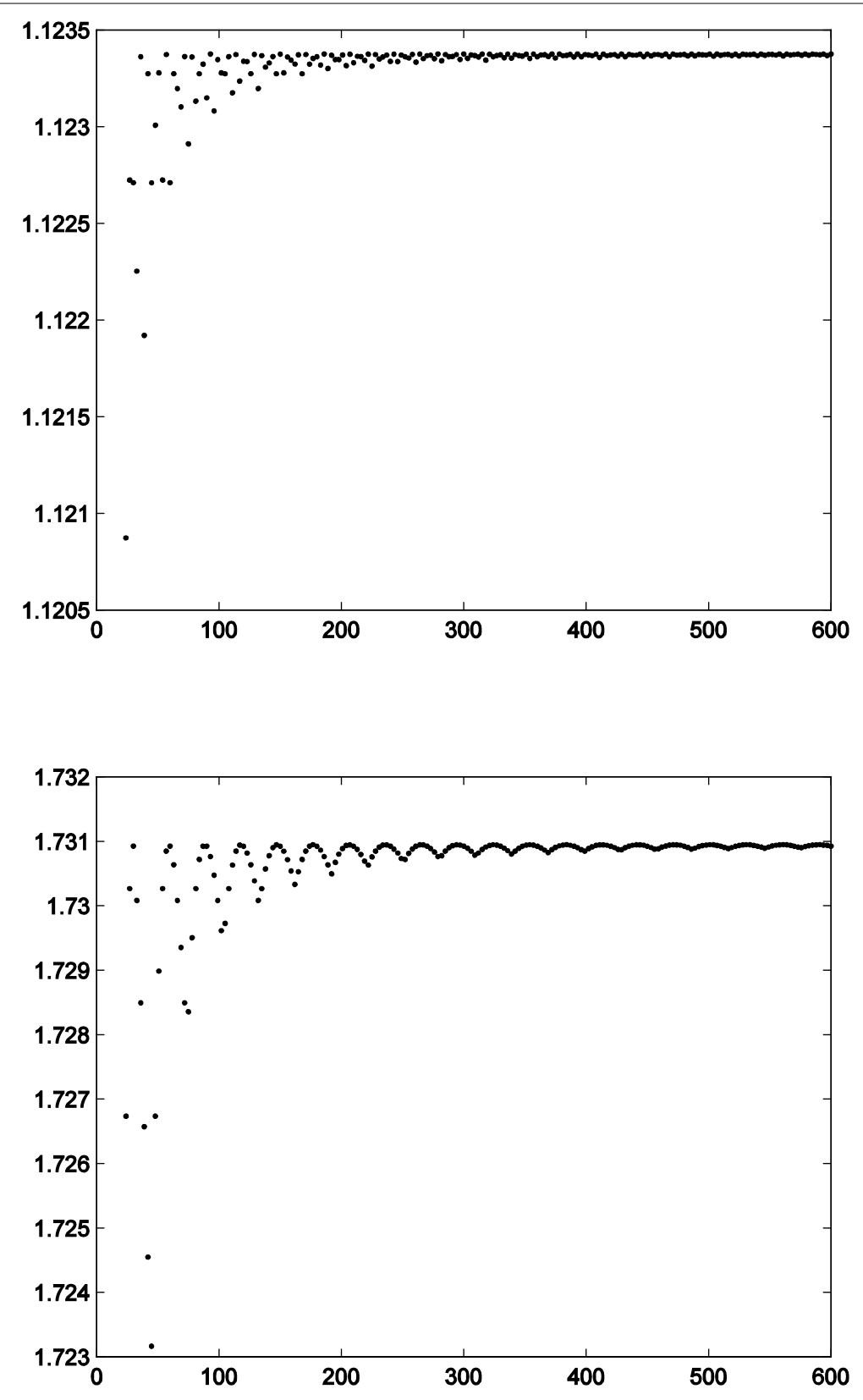

Figure 1 The spectral radius of $M M^{T}$ and $\widetilde{M} \tilde{M}^{T}$, respectively (from top to bottom)

$$
\begin{aligned}
\widetilde{h}_{1} \approx & {[-0.0047182543,-0.0021152562,0.0030907400,0.038680809,0.033766352,} \\
& 0.016128444,-0.75722871,1.3447918], \\
\widetilde{h}_{2} \approx & {[0.14107656,0.063246501,-0.092413623,-0.45441064,-0.69483538,} \\
& -0.94219467,4.8815912,0] / 4
\end{aligned}
$$

(whereas the other half is symmetric/antisymmetric and so skipped). See Fig. 1 for the spectral radius of $M_{n} M_{n}^{T}$ and $\widetilde{M}_{n} \widetilde{M}_{n}^{T}$. From Theorem 2.3 , we can obtain $\|T\| \approx 1.06$, which 


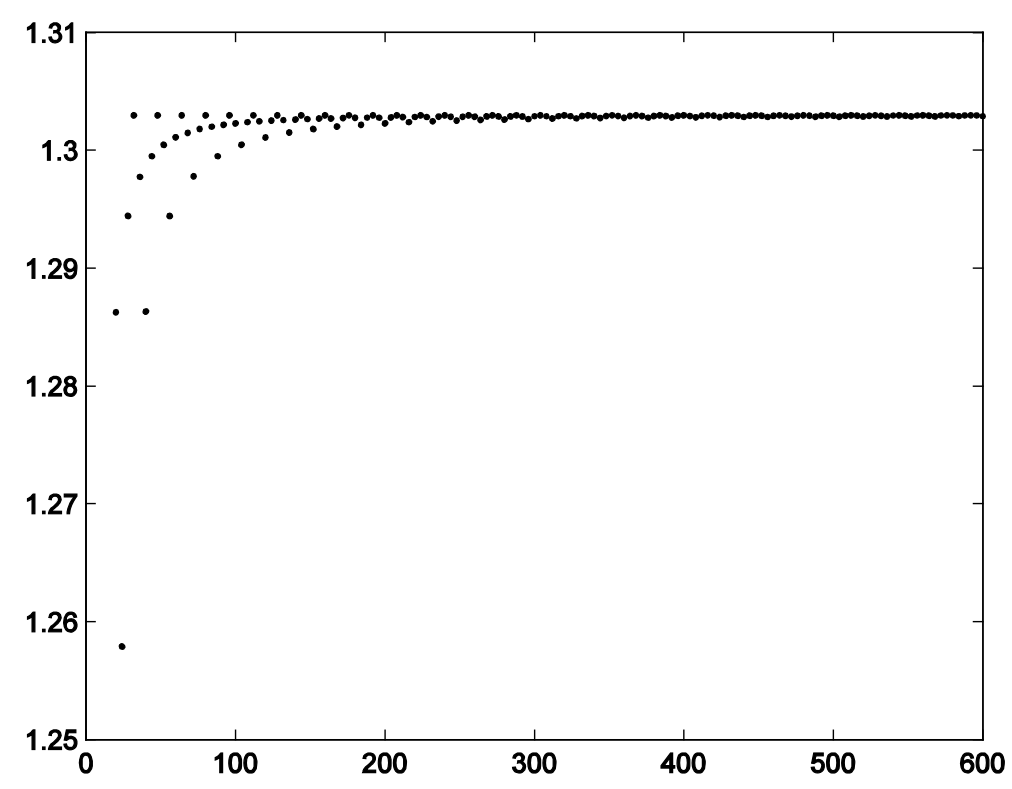

Figure 2 The spectral radius of $M M^{\top}\left(\tilde{M} \tilde{M}^{T}\right)$

is an approximation and not an exact value. Similarly, $\left\|T^{-1}\right\| \approx 1.32$. By Theorem 2.5 , we can calculate that $0.66 \leq\|T\| \leq 1.15$ and $0.94 \leq\left\|T^{-1}\right\| \leq 1.51$.

Example 3.2 In [20], a 4-band symmetric biorthogonal wavelets which is denoted as $\mathrm{Op}(12-12)$ is designed. The corresponding wavelet filter banks $\left\{h, g^{1}, g^{2}, g^{3}\right\}$ are as follows:

$$
\left[\begin{array}{cccccccccccc}
t_{0} & t_{1} & t_{2} & t_{3} & t_{4} & t_{5} & t_{5} & t_{4} & t_{3} & t_{2} & t_{1} & t_{0} \\
t_{1} & -t_{0} & -t_{3} & t_{2} & t_{5} & -t_{4} & -t_{4} & t_{5} & t_{2} & -t_{3} & -t_{0} & t_{1} \\
\widetilde{t}_{0} & -\widetilde{t}_{1} & \widetilde{t}_{2} & -\widetilde{t}_{3} & \widetilde{t}_{4} & -\widetilde{t}_{5} & \widetilde{t}_{5} & -\widetilde{t}_{4} & \widetilde{t}_{3} & -\widetilde{t}_{2} & \widetilde{t}_{1} & -\widetilde{t}_{0} \\
\widetilde{t}_{1} & \widetilde{t}_{0} & -\widetilde{t}_{3} & -\widetilde{t}_{2} & \widetilde{t}_{5} & \widetilde{t}_{4} & -\widetilde{t}_{4} & -\widetilde{t}_{5} & \widetilde{t}_{2} & \widetilde{t}_{3} & -\widetilde{t}_{0} & -\widetilde{t}_{1}
\end{array}\right],
$$

where $t_{0}=0.01129264, t_{1}=-0.01660958, t_{2}=-0.01418315, t_{3}=0.02102888, t_{4}=$ $0.4676785, t_{5}=0.5307927, \widetilde{t}_{0}=-0.07653, \widetilde{t}_{1}=-0.04528, \widetilde{t}_{2}=0.01722, \widetilde{t}_{3}=0.11097$, $\tilde{t}_{4}=0.46556, \widetilde{t}_{5}=0.52806$.

It has been shown that the eigenvalues of $M_{4 n}^{T} M_{4 n}$ appear in pairs of reciprocal, $M_{4 n}^{T} M_{4 n}$ and $\widetilde{M}_{4 n}^{T} \widetilde{M}_{4 n}$ have the same eigenvalues [20]. It is obvious that $\max \left\{\lambda_{i}\right\}=\max \left\{\widetilde{\lambda}_{i}\right\}=\frac{1}{\min \left\{\lambda_{i}\right\}}=$ $\frac{1}{\min \left\{\tilde{\lambda}_{i}\right\}}$. See Fig. 2 for the spectral radius of $M_{n} M_{n}^{T}$ and $\widetilde{M}_{n} \widetilde{M}_{n}^{T}$. From Theorem 2.3, we can obtain $\|T\| \approx 1.14$, which is an approximation and not an exact value. According to Theorem 2.5, we have $\|T\|=\left\|T^{-1}\right\| \leq 1.18$.

\section{Conclusions and future work}

We can obtain the upper bound and the lower bound or an approximation of the sub-band operator's norm based on the theory of circular matrix which plays an important role. We will calculate their norm for some special symmetric wavelets and design a family of biorthogonal wavelets based on the size of the norm. 


\section{Acknowledgements}

The authors would like to thank the editors and reviewers for their valuable comments, which greatly improved the readability of this paper. This work supported by the Natural Science Foundation of Hunan Province (No. 16JJ6102), the Outstanding Youth Foundation of Hunan Province Department of Education (No. 17B182), the Doctoral Foundation of Hunan University of Arts and Science (No. 15BSQD02), the College Students Research Study and Innovative Experiment Project of Hunan Province (No. 17541).

\section{Competing interests}

The authors declare that they have no competing interests.

\section{Authors' contributions}

All authors contributed to each part of this work equally and read and approved the final manuscript.

\section{Author details}

'Hunan Province Cooperative Innovation Center for The Construction Development of Dongting Lake Ecological Economic Zone, Hunan University of Arts and Science, Changde, P.R. China. ${ }^{2}$ College of Mathematics and Computer Science, Hunan Normal University, Changsha, P.R. China.

\section{Publisher's Note}

Springer Nature remains neutral with regard to jurisdictional claims in published maps and institutional affiliations.

Received: 11 October 2017 Accepted: 3 February 2018 Published online: 17 February 2018

\section{References}

1. Daubechies, I., Han, B., Ron, A., Shen, Z.: Framelets, MRA-based constructions of wavelet frames. Appl. Comput. Harmon. Anal. 14, 1-46 (2003)

2. Gavruta, L.: Frames for operators. Appl. Comput. Harmon. Anal. 32, 139-144 (2012)

3. Sun, W., Zhou, X.: Density and stability of wavelet frames. Appl. Comput. Harmon. Anal. 15, 117-133 (2003)

4. Heil, C., Kutyniok, G.: Density of wavelet frames. J. Geom. Anal. 13, 479-493 (2003)

5. Kim, H., Kim, R., Lee, Y., Yoon, J.: Quasi-interpolatory refinable functions and construction of biorthogonal wavelet systems. Adv. Comput. Math. 33, 255-283 (2010)

6. Cohen, A., Daubechies, I., Feauveau, J.: Biorthogonal bases of compactly supported wavelets. Commun. Pure Appl. Math. 45, 485-560 (1992)

7. Didenko, V.: Spectral radii of refinement and subdivision operators. Proc. Am. Math. Soc. 33, 2335-2346 (2005)

8. Han, B.: Symmetric orthonormal scaling functions and wavelets with dilation factor 4. Adv. Comput. Math. 8, 221-247 (1998)

9. Bi, N., Dai, X., Sun, Q.: Construction of compactly supported M-band wavelets. Appl. Comput. Harmon. Anal. 6, 113-131 (1999)

10. Chui, C., Lian, J.: Construction of compactly supported symmetric and antisymmetric orthonormal wavelets with scale $=3$. Appl. Comput. Harmon. Anal. 2, 68-84 (1995)

11. Zou, Q., Wang, G., Yang, M.: Spectral radius of biorthogonal wavelets with its application. J. Korean Math. Soc. 51, 941-953 (2014)

12. Wang, G., Zou, Q., Yang, M.: Sub-band operators and saddle point wavelets. Appl. Math. Comput. 227, 27-42 (2014)

13. Cui, L., Zhai, B., Zhang, T.: Existence and design of biorthogonal matrix-valued wavelets. Nonlinear Anal., Real World Appl. 10, 2679-2687 (2009)

14. Jiang, Q.: Biorthogonal wavelets with 4-fold axial symmetry for quadrilateral surface multiresolution processing. Adv. Comput. Math. 34, 127-165 (2011)

15. He, T.: Biorthogonal wavelets with certain regularities. Appl. Comput. Harmon. Anal. 11, 227-242 (2001)

16. Zhuang, X.: Matrix extension with symmetry and construction of biorthogonal multiwavelets with any integer dilation. Appl. Comput. Harmon. Anal. 33, 159-181 (2012)

17. Chui, C., Shi, X.: Inequalities of Littlewood-Paley type for frames and wavelets. SIAM J. Math. Anal. 24, 263-277 (1993)

18. Naylor, A., Sell, G.: Linear Operator Theory in Engineering and Science. Springer, New York (2000)

19. Wang, G.: Four-bank compactly supported bi-symmetric orthonormal wavelets bases. Opt. Eng. 43, 2362-2368 (2004)

20. Zou, Q., Wang, G.: Optimal model for 4-band biorthogonal wavelets bases for fast calculation. J. Inequal. Appl. 2017, Article ID 222 (2017)

21. Zou, Q., Wang, G.: Construction of biorthogonal 3-band wavelets with symmetry and high vanishing moments. J. Math. 35, 12-22 (2015) 